\title{
Non-perturbative scale evolution of four-fermion operators in two-flavour QCD
}

\section{$\bar{A} L P H A$ \\ Collaboration}

\section{Petros Dimopoulos, Gregorio Herdoiza*, Anastassios Vladikas}

INFN, Sezione di Roma Tor Vergata

and Dipartimento di Fisica, Università di Roma "Tor Vergata"

Via della Ricerca Scientifica 1, I-00133 Rome, Italy

E-mail: \{dimopoulos, herdoiza, vladikas\}eroma2.infn.it

\section{Filippo Palombi}

Institut für Kernphysik, Johannes Gutenberg Universität

Johann Joachim Becher-Weg 45, D-55099 Mainz, Germany

E-mail: palombi@kph.uni-mainz.de

\section{Carlos Pena}

CERN, Physics Department, TH Division, CH-1211 Geneva 23, Switzerland

E-mail: Carlos.pena.ruano@cern.ch

\section{Stefan Sint}

School of Mathematics, Trinity College, Dublin 2, Ireland

E-mail: sintemaths.tcd.ie

\begin{abstract}
We apply finite-size recursion techniques based on the Schrödinger functional formalism to determine the renormalization group running of four-fermion operators which appear in the $\Delta S=2$ effective weak Hamiltonian of the Standard Model. Our calculations are done using $\mathscr{O}(a)$ improved Wilson fermions with $N_{\mathrm{f}}=2$ dynamical flavours. Preliminary results are presented for the four-fermion operator which determines the $B_{K}$-parameter in tmQCD.
\end{abstract}

XXIVth International Symposium on Lattice Field Theory

July 23-28, 2006

Tucson, Arizona, USA

\footnotetext{
*Speaker.
} 


\section{Introduction}

A precise determination of the Kaon $B$-parameter is required to constrain the CKM unitarity triangle analysis. $B_{K}$ is defined in terms of hadronic matrix elements which can be computed using lattice QCD. At present, the inclusion of dynamical quark effects is an essential requirement in these lattice calculations. Indeed, a remarkably good agreement has been found between several independent quenched determinations of $B_{K}$ [1], leaving the "quenching" effects as the largest uncertainty. A complete study of the systematic effects, other than quenching, has been performed in ref. [2] and reviewed in this conference [3]. There are indications that once dynamical quarks are taken into account, a major source of uncertainty on lattice results arises from the determination of the renormalization factors of the four-fermion operators [1]. In principle, this uncertainty can be completely eliminated by a non-perturbative renormalization procedure. Similarly, the renormalization group (RG) running of the operator from hadronic scales up to high-energies, where perturbation theory can be safely applied, is best performed non-perturbatively. Here we report on the status of such a non-perturbative renormalization using $N_{\mathrm{f}}=2$ dynamical flavours. In particular, we present preliminary results for the scale evolution of the four-fermion operator relevant for the determination of $B_{K}$ in the context of tmQCD [ [

The theoretical description of the $K^{0}-\bar{K}^{0}$ oscillation is controlled, once high-energy scales are integrated through an operator product expansion procedure, by the matrix element $\left\langle\bar{K}^{0}\left|O^{\Delta S=2}\right| K^{0}\right\rangle$, where the four-fermion operator is defined as follows:

$$
O^{\Delta S=2} \equiv\left[\bar{s} \gamma_{\mu}\left(1-\gamma_{5}\right) d\right]\left[\bar{s} \gamma_{\mu}\left(1-\gamma_{5}\right) d\right]=O_{\mathrm{VV}+\mathrm{AA}}-O_{\mathrm{VA}+\mathrm{AV}} .
$$

The strange and down quark fields are denoted by $s$ and $d$ respectively. The $B_{K}$-parameter is expressed in terms of the parity-even $O_{\mathrm{vv}+\mathrm{AA}}$ operator :

$$
B_{K} \equiv \frac{\left\langle\bar{K}^{0}\left|O_{\mathrm{vV}+\mathrm{AA}}\right| K^{0}\right\rangle}{\frac{8}{3} F_{K}^{2} m_{K}^{2}} .
$$

Parity conservation ensures that the matrix element $\left\langle\bar{K}^{0}\left|O_{\mathrm{vA}+\mathrm{AV}}\right| K^{0}\right\rangle$, involving the parity-odd operator, is identically zero.

In lattice regularizations preserving chiral symmetry the operator $O_{\mathrm{VV}+\mathrm{AA}}$ is multiplicatively renormalizable. This is not the case for Wilson fermions because in this case chiral symmetry is broken at non-zero lattice spacing. The renormalization of $O_{\mathrm{VV}+\mathrm{AA}}$ is therefore more involved since mixing with four other dimension- 6 operators has to be considered. On the other hand, discrete symmetries protect the parity-odd operator $O_{\mathrm{VA}+\mathrm{AV}}$, so as to preserve multiplicative renormalization also in the case of Wilson fermions [5, 6].

The inclusion of a "twisted mass" term in the fermionic action opens the way to improve the renormalization properties of Wilson fermions. The twisted-mass theory is related to standard QCD through an axial transformation of the quarks fields. By choosing appropriate formulations of tmQCD (see ref. [2] for two of these formulations), it is possible to relate the QCD $O_{\mathrm{vV}+\mathrm{AA}}$ operator to a partner $O_{\mathrm{VA}+\mathrm{AV}}$ in tmQCD where the property of multiplicative renormalization of this operator is preserved. This method allows the determination of $B_{K}$ only via multiplicative renormalization, thus avoiding mixing with operators of wrong chirality. Another proposal in this direction uses chiral Ward-Takahashi identities to relate parity-odd and parity-even operators [7]. 
Our strategy to renormalize the operator $O_{\mathrm{VA}+\mathrm{AV}}$ closely follows the one used in ref. [8] for the quenched case. The connection between the renormalization group invariant operator $\hat{O}_{\mathrm{VA}+\mathrm{AV}}$ and its bare counterpart $O_{\mathrm{VA}+\mathrm{AV}}\left(g_{0}\right)$ can be written in the following way:

$$
\hat{O}_{\mathrm{VA}+\mathrm{AV}}(x)=\lim _{g_{0} \rightarrow 0} \mathscr{Z}_{\mathrm{VA}+\mathrm{AV} ; \mathrm{s}}\left(g_{0}\right) O_{\mathrm{VA}+\mathrm{AV}}\left(x ; g_{0}\right) .
$$

The RGI operator is independent of the renormalization scheme and scale when the renormalization conditions are imposed at zero quark mass [9]. The renormalization factor $\mathscr{Z}_{\mathrm{VA}+\mathrm{AV} ; \mathrm{s}}\left(g_{0}\right)$ is scale-independent but depends on the scheme $s$ (only through cutoff effects) and on the lattice regularization. These dependences are manifest when decomposing $\mathscr{Z}_{\mathrm{VA}+\mathrm{AV} ; \mathrm{s}}\left(g_{0}\right)$ into:

$$
\mathscr{Z}_{\mathrm{VA}+\mathrm{AV} ; \mathrm{s}}\left(g_{0}\right)=\hat{Z}_{\mathrm{VA}+\mathrm{AV} ; \mathrm{s}}\left(\mu_{\mathrm{min}}\right) Z_{\mathrm{VA}+\mathrm{AV} ; \mathrm{s}}\left(g_{0}, a \mu_{\mathrm{min}}\right) .
$$

The first factor on the r.h.s. of eq. (1.4) controls the RG-running of the operator from the reference scale $\mu_{\min }$ to an infinite scale. It is independent of the regularisation. The second factor, $Z_{\mathrm{VA}+\mathrm{AV} ; \mathrm{s}}\left(g_{0}, a \mu_{\mathrm{min}}\right)$, relates the bare lattice operator to its continuum value at the hadronic scale $\mu_{\min }$. This factor is therefore dependent on both the regularization and the scale. Both factors on the r.h.s. of eq. (1.4) depend on the renormalization scheme. In this report, we focus on the more expensive part of the renormalization procedure, that is, the computation of the contribution of the non-perturbative evolution function $\hat{Z}_{\mathrm{VA}+\mathrm{AV} ; \mathrm{s}}\left(\mu_{\min }\right)$ describing the running in the scale range $1 \mathrm{GeV}-100 \mathrm{GeV}$.

\section{Renormalization group running of four-fermion operators}

Let us first define a renormalized four-fermion operator $O_{R}$ at a reference scale $\mu$ :

$$
O_{R}(x ; \mu)=\lim _{a \rightarrow 0} Z_{O}\left(g_{0}, a \mu\right) O\left(x ; g_{0}\right) .
$$

The running of the renormalized operator $O_{R}(\mu)$ is controlled by its anomalous dimension $\gamma_{O}(\bar{g})$, defined as :

$$
\mu \frac{\partial}{\partial \mu} O_{R}(x ; \mu)=\gamma_{O}(\bar{g}) O_{R}(x ; \mu) .
$$

In mass-independent renormalization schemes [ [ 9 ] as those we consider here, the function $\gamma_{O}(\bar{g})$ only depends on the renormalized coupling $\bar{g}$. The perturbative expansion of $\gamma_{O}(\bar{g})$ is given by

$$
\gamma_{O}(g) \stackrel{g \rightarrow 0}{\sim}-g^{2}\left(\gamma_{O}^{(0)}+\gamma_{O}^{(1)} g^{2}+\gamma_{O}^{(2)} g^{4}+\ldots\right)
$$

with $\gamma_{O}^{(0)}$ a universal coefficient. By combining eqs. (2.1) and (2.2), it is possible to relate the anomalous dimension of $O_{R}$ to its scale-dependent renormalization factor :

$$
\gamma_{O}(\bar{g}(\mu))=\lim _{a \rightarrow 0}\left(\mu \frac{\partial}{\partial \mu} Z_{O}\left(g_{0}, a \mu\right)\right) Z_{O}\left(g_{0}, a \mu\right)^{-1} .
$$

The RGI operator is obtained upon the formal integration of eq. (2.2). It is given by

$$
\hat{O}(x)=O_{R}(x ; \mu)\left[\frac{\bar{g}^{2}(\mu)}{4 \pi}\right]^{-\gamma_{O}^{(0)} /\left(2 b_{0}\right)} \exp \left\{-\int_{0}^{\bar{g}(\mu)} d g\left(\frac{\gamma_{O}(g)}{\beta(g)}-\frac{\gamma_{O}^{(0)}}{b_{0} g}\right)\right\} .
$$


The integral in the r.h.s of eq. (2.5) describes the scale evolution of $\gamma_{O}(\bar{g})$. The evolution function of the operator $O_{R}$ between the renormalization scale $\mu$ and an arbitrary scale $\mu^{\prime}$ is given by:

$$
U\left(\mu^{\prime}, \mu\right) \equiv \exp \left\{\int_{\bar{g}(\mu)}^{\bar{g}\left(\mu^{\prime}\right)} \frac{\gamma_{O}(g)}{\beta(g)} \mathrm{d} g\right\}=\lim _{a \rightarrow 0} \frac{Z_{O}\left(g_{0}, a \mu^{\prime}\right)}{Z_{O}\left(g_{0}, a \mu\right)} .
$$

The running of the renormalized four-fermion operator $O_{R}$ can therefore be performed by constructing ratios of the renormalization factors $Z_{O}$ at different scales.

Our renormalization schemes are defined in the Schrödinger functional (SF) formalism. This technique has been used to determine the scale evolution of physical quantities such as the strong coupling [10, 11] or the quark mass [12, 13]. These studies were performed both with and without dynamical quarks. In the case of four-fermion operators [8] the running was carried out in the quenched approximation. We regularize the theory on a lattice of physical size $L^{4}$ using standard SF boundary conditions allowing to carry out simulations at zero quark masses. The SF is used as a mass-independent renormalization scheme; since the renormalization factors are flavourindependent, they can also be used to renormalize the $B$-parameters in the Kaon, $D$ and $B$-meson sectors. The renormalization conditions are imposed at a scale $\mu$ equal to the IR cutoff $1 / L$.

Let us now concentrate on the case of the local parity-odd four-fermion operator:

$$
O_{\mathrm{VA}+\mathrm{AV}}(x)=\frac{1}{2}\left[\left(\bar{\psi}_{1} \gamma_{\mu} \psi_{2}\right)\left(\bar{\psi}_{3} \gamma_{\mu} \gamma_{5} \psi_{4}\right)+\left(\bar{\psi}_{1} \gamma_{\mu} \gamma_{5} \psi_{2}\right)\left(\bar{\psi}_{3} \gamma_{\mu} \psi_{4}\right)+\left(\psi_{2} \leftrightarrow \psi_{4}\right)\right] .
$$

Four distinct valence flavours are used in the definition of the operator. The SF correlation functions used to extract the operator $O_{\mathrm{vA}+\mathrm{AV}}(x)$ are:

$$
\mathscr{F}_{\left[\Gamma_{\mathrm{A}}, \Gamma_{\mathrm{B}}, \Gamma_{\mathrm{C}}\right]}\left(x_{0}\right)=\frac{1}{L^{3}}\left\langle\mathscr{O}_{21}\left[\Gamma_{\mathrm{A}}\right] \mathscr{O}_{45}\left[\Gamma_{\mathrm{B}}\right] O_{\mathrm{VA}+\mathrm{AV}}(x) \mathscr{O}_{53}^{\prime}\left[\Gamma_{\mathrm{C}}\right]\right\rangle
$$

where $\mathscr{O}$ and $\mathscr{O}^{\prime}$ are the interpolating fields on the time boundaries (refer to [队] for a full explanation of the notations). Several choices of the Dirac matrices $\Gamma_{\mathrm{A}, \mathrm{B}, \mathrm{C}}$ are allowed. We will focus here on the particular choice $\Gamma_{\mathrm{A}}=\Gamma_{\mathrm{B}}=\Gamma_{\mathrm{C}}=\gamma_{5}$. Note that a "spectator" valence quark $\psi_{5}$ is used in eq. (2.8). It is useful to keep in mind that since the quarks are massless in this mass-independent renormalization scheme, flavour only enters through Wick contractions.

The logarithmic divergences of the local operator $O_{\mathrm{VA}+\mathrm{AV}}(x)$ are isolated by dividing out from the correlator $\mathscr{F}$ the divergences coming from the boundaries and the external legs. This is obtained through the ratio:

$$
h\left(x_{0}\right)=\frac{\mathscr{F}\left[\gamma_{5}, \gamma_{5}, \gamma_{5}\right]\left(x_{0}\right)}{f_{1}^{3 / 2}},
$$

where $f_{1}$ is the boundary-to-boundary correlation function : $f_{1}=-1 /\left(2 L^{6}\right)\left\langle\mathscr{O}_{12}^{\prime}\left[\gamma_{5}\right] \mathscr{O}_{21}\left[\gamma_{5}\right]\right\rangle$. The renormalized ratio $h_{R}$ can be written as follows:

$$
h_{\mathrm{R}}\left(x_{0} ; \mu\right)=Z_{\mathrm{VA}+\mathrm{AV}}\left(g_{0}, a \mu\right) h\left(x_{0} ; g_{0}\right) \text {, }
$$

where the renormalization factor is fixed by imposing the renormalization condition :

$$
Z_{\mathrm{VA}+\mathrm{AV}}\left(g_{0}, a \mu=1 / L\right) h\left(x_{0}=L / 2 ; g_{0}\right)=\left.h_{\mathrm{s}}\left(x_{0}=L / 2 ; g_{0}\right)\right|_{g_{0}=0},
$$


i.e. at tree level $Z_{\mathrm{VA}+\mathrm{AV}}=1$. The renormalization condition is taken at the scale $\mu=1 / L$ and therefore at fixed renormalized coupling $u=\bar{g}^{2}(1 / L)$. Note that as the quarks are massless, once the continuum limit $a \rightarrow 0$ is taken, the only remaining scale is $L$.

The running of the scale-dependent factor $Z_{\mathrm{VA}+\mathrm{AV}}\left(g_{0}, a \mu\right)$ is implemented in the SF formalism via the step scaling function (SSF), defined in the continuum as:

$$
\sigma_{\mathrm{VA}+\mathrm{AV}}(u)=\lim _{a \rightarrow 0} \Sigma_{\mathrm{VA}+\mathrm{AV}}(u, a / L), \quad \Sigma_{\mathrm{VA}+\mathrm{AV}}(u, a / L)=\left.\frac{Z_{\mathrm{VA}+\mathrm{AV}}\left(g_{0}, a / 2 L\right)}{Z_{\mathrm{VA}+\mathrm{AV}}\left(g_{0}, a / L\right)}\right|_{m=0, \bar{g}^{2}(1 / L)=u} .
$$

The SSF can be written in terms of the evolution function $U: \sigma_{\mathrm{VA}+\mathrm{AV}}(u)=U_{\mathrm{VA}+\mathrm{AV}}(1 / 2 L, 1 / L)$. The $\mathrm{SSF}$ is used to run the operator $O_{\mathrm{VA}+\mathrm{AV}}$ between two scales differing by a factor of two. By iterating this procedure the running of the operator can be performed over a large range of scales.

\section{Non-perturbative study of the step scaling function}

The computation of the SSF is performed with $N_{\mathrm{f}}=2$ flavours of $\mathscr{O}(a)$ improved Wilson fermions. We evaluate $\Sigma_{\mathrm{VA}+\mathrm{AV}}(u, a / L)$ at six values of the renormalized coupling $u$ (labelled, in increasing order, $u 1, \ldots, u 6)$. At each of these couplings, we consider three lattice resolutions $L / a=$ $6,8,12$ to extrapolate our data to the continuum limit. The unquenched configurations employed in our computation have been previously used in the study of the quark mass renormalization [13] (the description of our simulation setup can be found in this reference). In fig.1] we present the status of the ongoing determination of $\Sigma_{\mathrm{VA}+\mathrm{AV}}(u, a / L)$. Data for some of the simulation points has not yet been included as it is still being generated. Moreover, the statistical errors of our preliminary data in fig.11 will reduce when the complete set of configurations will be considered. The integrated autocorrelation times are included in the error estimate. We observe that the autocorrelations grow when increasing the coupling and when approaching the continuum limit.

The $\mathscr{O}(a)$ improvement of the dimension-six operator $O_{\mathrm{VA}+\mathrm{AV}}$ has not been implemented. Although a Symanzik improvement program is possible, the mixing of $O_{\mathrm{VA}+\mathrm{AV}}$ with several dimensionseven operators makes it unpractical. For each of the couplings $u$, the continuum limit of $\Sigma_{\mathrm{VA}+\mathrm{AV}}(u, a / L)$ should therefore be taken through a linear extrapolation. This is illustrated in fig.11 in those cases where three $L / a$ resolutions are already available. As our data seems to show rather small cutoff effects, we have also tried to fit $\Sigma_{\mathrm{VA}+\mathrm{AV}}(u, a / L)$ to a constant (we perform a weighted average and, somehow abusively, we refer to it as a "constant fit"). In the case of the couplings $u 1, u 3, u 5$ and $u 6$ this fit was performed by discarding the $L / a=6$ data which, being far from the continuum, is expected to contain large cutoff effects. By comparing the linear and the constant fit, we observe good agreement of the extrapolated values. A more refined analysis of the continuum extrapolation will be undertaken once our complete set of data will be available.

In a tentative study of the quality of our data, we present in fig. 2 the $\operatorname{SSF} \sigma_{\mathrm{VA}+\mathrm{AV}}(u)$. The $N_{\mathrm{f}}=2$ data is compared to the quenched one from ref. [8]. The same renormalization scheme and fitting procedures is used in both the $N_{\mathrm{f}}=2$ and $N_{\mathrm{f}}=0$ data (in particular, also in the quenched case we perform a constant fit to the continuum). ${ }^{1}$ In the strong coupling regime, $u \sim 3.5$, we observe

\footnotetext{
${ }^{1}$ Note that this comparison is only intended to study the quality of our data. It is indeed improper to compare $N_{\mathrm{f}}=2$ and quenched physical results at this stage since the respective renormalized couplings are not taken at the same physical scale.
} 

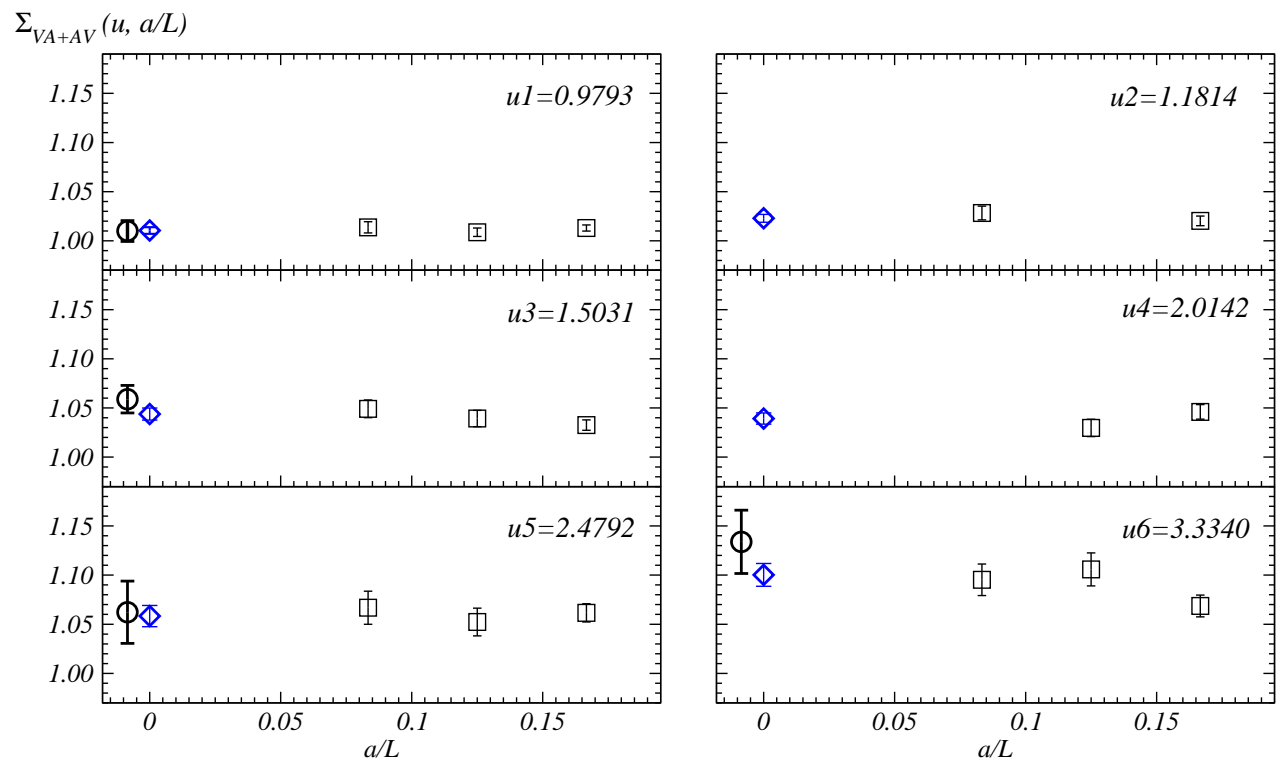

Figure 1: Continuum extrapolation of the $\operatorname{SSF} \Sigma_{\mathrm{VA}+\mathrm{AV}}(u, a / L)$ at fixed renormalized couplings $u$. The empty circles correspond to the extrapolated value obtained through a linear fit and the diamond to the one obtained via a fit to a constant. Results are preliminary.

a similar pattern in the $N_{\mathrm{f}}=2$ and $N_{\mathrm{f}}=0$ data when comparing the value of $\sigma_{\mathrm{VA}+\mathrm{AV}}(u)$ obtained through a linear and a constant extrapolation to the continuum: in both $N_{\mathrm{f}}=0,2$ cases the linear extrapolation points lie above the constant-fit ones. Due to large statistical errors, the $N_{\mathrm{f}}=2$ data shows a better agreement between linear and constant fit. In fig. 2, we also plot the perturbative expressions of $\sigma_{\mathrm{VA}+\mathrm{AV}}(u)$ in both the $N_{\mathrm{f}}=2$ and $N_{\mathrm{f}}=0$ cases. These expressions were computed at next-to-leading order (NLO) in ref. [14]. In our chosen renormalization scheme, we observe a fairly good agreement between the perturbative curve and the non-perturbative data in the small coupling region and some signs of deviations in the strong coupling regime. We have considered nine different renormalization schemes (for the definitions of these schemes, refer to [8, 14]). The empirical criterion to identify the more appropriate schemes is to consider those having a small NLO term, of the same sign as the LO one, in the perturbative expansion of $\sigma_{\mathrm{VA}+\mathrm{AV}}(u)$. We have checked that our best available scheme is indeed the one of eq. (2.9).

\section{Conclusions}

We have presented the status of the computation of the non-perturbative RG running of the four-fermion operator $O_{\mathrm{VA}+\mathrm{AV}}$ using lattice QCD with two dynamical quarks. This calculation will soon allow us to determine the universal renormalization factor $\hat{Z}_{\mathrm{VA}+\mathrm{AV} ; \mathrm{s}}(\mu)$ in eq. (1.4). The second factor on the r.h.s of this equation is simpler to determine, compared to $\hat{Z}_{\mathrm{VA}+\mathrm{AV} ; \mathrm{s}}(\mu)$, as it depends only on a single scale $\mu$. This determination will allow to complete the renormalization of the operator $O_{\mathrm{VA}+\mathrm{AV}}$. As our renormalization scheme is flavour-independent, the same renormalization factors can be used to determine the $B$-parameters in the strange, charm and beauty sectors. 


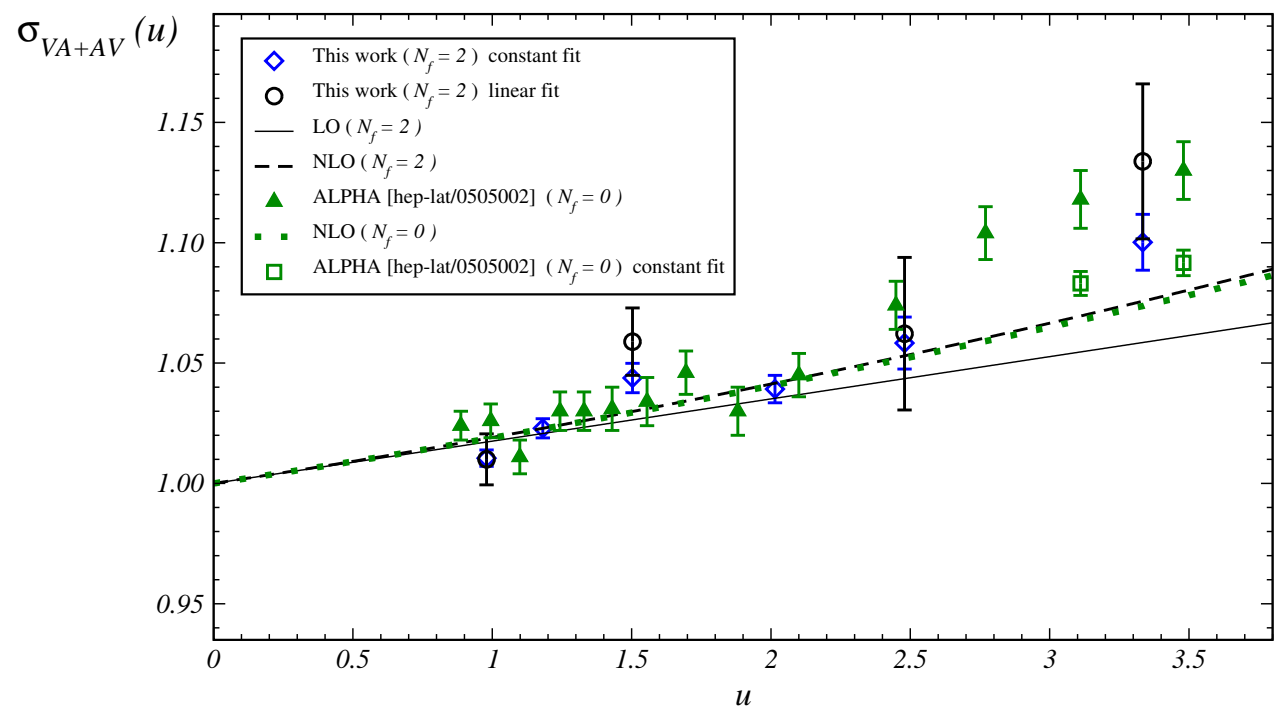

Figure 2: The step scaling function $\sigma_{\mathrm{VA}+\mathrm{AV}}(u)$ (discrete points) is compared to the LO and NLO perturbative results. In order to evaluate the quality of our data we compare our $N_{\mathrm{f}}=2$ results with quenched data from ref. [8]. Results are preliminary.

\section{Acknowledgements}

We thank Roberto Frezzotti and Francesco Knechtli for useful discussions. We would specially like to thank Michele Della Morte for help and advice in the first stages of this work. We also thank the Computer Center of DESY-Zeuthen for their support.

\section{References}

[1] C. Dawson, PoS LAT2005 (2006) 007

[2] P. Dimopoulos et al., [ALPHA Collaboration], Nucl. Phys. B 749 (2006) 69 [hep-ph/0601002].

[3] C. Pena, "Twisted mass QCD for weak matrix elements," PoS LAT2006 (2006) 019

[4] R. Frezzotti et al., JHEP 0108 (2001) 058 [hep-lat/0101001].

[5] C. W. Bernard, T. Draper, G. Hockney and A. Soni, Nucl. Phys. Proc. Suppl. 4 (1988) 483

[6] A. Donini et al., Eur. Phys. J. C 10 (1999) 121 [hep-lat/9902030].

[7] D. Becirevic et al., Phys. Lett. B 487 (2000) 74 [hep-lat/0005013].

[8] M. Guagnelli et al., [ALPHA Collaboration], JHEP 0603 (2006) 088 [hep-lat/0505002].

[9] S. Weinberg, Phys. Rev. D8 (1973) 3497.

[10] M. Lüscher, R. Sommer, P. Weisz and U. Wolff, Nucl. Phys. B 413 (1994) 481 [hep-lat/9309005].

[11] M. Della Morte et al., [ALPHA Collaboration], Nucl. Phys. B 713 (2005) 378 [hep-lat/0411025].

[12] S. Capitani et al., [ALPHA Collaboration], Nucl. Phys. B 544 (1999) 669 [hep-lat/9810063].

[13] M. Della Morte et al., [ALPHA Collaboration], Nucl. Phys. B 729 (2005) 117 [hep-lat/0507035].

[14] F. Palombi, C. Pena and S. Sint, JHEP 0603 (2006) 089 [hep-lat/0505003]. 